\title{
CLOSING THE LOOP WITH FRACTAL INTERPOLATING FUNCTIONS FOR GEOPHYSICAL ENCODING
}

\author{
HUAI-HSIEN HUANG, ${ }^{*}$ CARLOS E. PUENTE,,$\S$ \\ ANDREA CORTIS $^{\dagger}$ and BELLIE SIVAKUMAR ${ }^{*, \ddagger}$ \\ ${ }^{*}$ Land, Air and Water Resources \\ University of California, Davis \\ One Shields Ave, Davis, CA 95616, USA \\ ${ }^{\dagger} I O N$ GX Technology \\ Houston, TX 77042, USA \\ ${ }^{\ddagger}$ Department of Civil and Environmental Engineering \\ University of New South Wales \\ Sydney, NSW 2052, Australia \\ §cepuente@ucdavis.edu
}

Received March 29, 2012

Accepted October 5, 2012

Published November 19, 2012

\begin{abstract}
Natural data sets, such as precipitation records, often contain geometries that are too complex to model in their totality with classical stochastic methods. In the past years, we have developed a promising deterministic geometric procedure, the fractal-multifractal (FM) method, capable of generating patterns as projections that share textures and other fine details of individual data sets, in addition to the usual statistics of interest. In this paper, we formulate an extension of the FM method around the concept of "closing the loop" by linking ends of two fractal
\end{abstract}

${ }^{\S}$ Corresponding author. 
interpolating functions and then test it on four geometrically distinct rainfall data sets to show that this generalization can provide excellent results.

Keywords: Fractals; Multifractals; Rainfall; Particle Swarm Optimization; Fractal-Multifractal Approach; Inverse Problem.

\section{INTRODUCTION}

Over the past few decades, research into the representation of complex natural data sets has resulted in the development of many sophisticated mathematical techniques, such as those based on stochastic theories and fractal geometry. ${ }^{1,2}$ Despite providing a suitable language for describing and simulating intricate data sets, these methods are still largely inadequate for studying the vast variety of time series on an individual basis. In particular, while relevant statistical (physical) attributes may be retained, the realizations given by these approaches often fail to replicate specific details (e.g. positions of major peaks) and textures (e.g. periods of no activity) of noisy and erratic data.

The intrinsic limitations with the above approaches inspired us to propose a fractal geometric methodology, ${ }^{3}$ called the fractal-multifractal (FM) approach, that uses fractal interpolating functions ${ }^{4}$ to transform multifractal measures into derived projections with the hopes of representing the overall complexity of natural patterns beyond key statistical features. Indeed, our FM approach (and its variants) is capable of deterministically generating a multitude of "seemingly random" patterns over one or more dimensions based on a small number of parameters yet still preserving distinct characteristics of data sets. ${ }^{3,5-10}$

In this paper, we introduce yet another generalization of our FM approach by combining projections of two fractal interpolating functions that share the same beginning and end points. The idea to "close the loop" was inspired by unrelated notions in particle physics, in particular, the necessity of loops to describe the weak gravitational forces using strings. The goodness of this idea is then tested with four distinct high-resolution rainfall data sets measured in Iowa City, USA.

\section{METHODS}

In this section, we describe the construction of the original fractal-multifractal approach and the new extension.

\subsection{The Original Fractal-Multifractal Approach}

In its original form, ${ }^{3}$ a FM pattern is obtained as the projection of the graph of a fractal interpolating function weighed by a multifractal measure. Specifically, the graph $G=\{(x, f(x)) \mid x \in[0,1]\}$ of such a function $f: x \rightarrow y$ passing by $N+1$ ordered points along $x,\left\{\left(x_{n}, y_{n}\right) \mid x_{0}<\cdots<x_{N}, n=0,1, \ldots, N\right\}$, is defined as the unique "attractor" of $N$ affine $\operatorname{maps}^{4}$ :

$$
\begin{array}{r}
w_{n}\left(\begin{array}{l}
x \\
y
\end{array}\right)=\left(\begin{array}{ll}
a_{n} & 0 \\
c_{n} & d_{n}
\end{array}\right)\left(\begin{array}{l}
x \\
y
\end{array}\right) \\
n=1, \ldots, N \\
n=1,
\end{array}
$$

where the vertical scalings $d_{n}$ satisfy $\left|d_{n}\right|<1$, and the other parameters $a_{n}, c_{n}, e_{n}$, and $f_{n}$ are given by the following initial conditions:

$$
w_{n}\left(\begin{array}{l}
x_{0} \\
y_{0}
\end{array}\right)=\left(\begin{array}{l}
x_{n-1} \\
y_{n-1}
\end{array}\right)
$$

and

$$
w_{n}\left(\begin{array}{l}
x_{N} \\
y_{N}
\end{array}\right)=\left(\begin{array}{l}
x_{n} \\
y_{n}
\end{array}\right),
$$

which contract the ends of the domain (in $x$ ) into interior subintervals.

In virtue of a fixed-point theorem, the attractor $G$ exists and contains the $N+1$ interpolating points. ${ }^{4}$ Substitution of Eq. (1) into (2) and (3) uniquely determines, in a linear fashion, the values of $a_{n}, c_{n}, e_{n}$, and $f_{n}$ in terms of $d_{n}$, the vertical scalings, and $\left(x_{n}, y_{n}\right)$, the coordinates of the interpolating points. Ultimately, the iteration of the affine mappings in Eq. (1) yields a unique (and hence deterministic) set $G$ having a fractal dimension $1 \leqslant D<2$.

In a practical setting, the graph of a fractal interpolating function, typically shaped as a convoluted wire, is obtained by a point wise sampling of the attractor through a procedure also known as the "chaos game." 4 The idea is to start the process at a given $\left(x_{n}, y_{n}\right)$ already in $G$ and progressively iterate the $N$ maps $w_{n}$ according to, for example, the 
outcomes of independent "coin tosses." As this process is performed, a unique invariant measure that reflects how the attractor is filled up is also induced over $G$. The existence of such a measure (akin to a histogram in practice) allows computing unique and again, fully deterministic - projections over the coordinates $x$ and $y$ (denoted by $d x$ and $d y$ ) that turn out to display irregular shapes as found in a variety of geophysical applications and beyond. ${ }^{5}$

Figure 1 shows an example of a fractal wire passing through the four points $\{(0,0),(0.26,1.93)$, $(0.53,-3.18),(1,1)\}$ as generated by $2^{14}$ iterations of the three maps

$$
\begin{aligned}
& w_{1}\left(\begin{array}{l}
x \\
y
\end{array}\right)=\left(\begin{array}{ll}
0.26 & 0 \\
1.93 & 0.01
\end{array}\right)\left(\begin{array}{l}
x \\
y
\end{array}\right)+\left(\begin{array}{l}
0 \\
0
\end{array}\right) \\
& w_{2}\left(\begin{array}{l}
x \\
y
\end{array}\right)=\left(\begin{array}{ll}
0.27 & 0 \\
-5.55 & 0.44
\end{array}\right)\left(\begin{array}{l}
x \\
y
\end{array}\right)+\left(\begin{array}{l}
0.26 \\
1.93
\end{array}\right)
\end{aligned}
$$

and

$$
w_{3}\left(\begin{array}{l}
x \\
y
\end{array}\right)=\left(\begin{array}{cc}
0.47 & 0 \\
4.99 & -0.82
\end{array}\right)\left(\begin{array}{l}
x \\
y
\end{array}\right)+\left(\begin{array}{r}
0.53 \\
-3.18
\end{array}\right)
$$

In other words, the maps $w_{1}, w_{2}$, and $w_{3}$ have contractive vertical scalings $d_{1}=0.01, d_{2}=0.44$, and $d_{3}=-0.82$ and operate into the intervals $[0,0.26]$, $[0.26,0.53]$, and $[0.53,1]$, respectively. In addition to
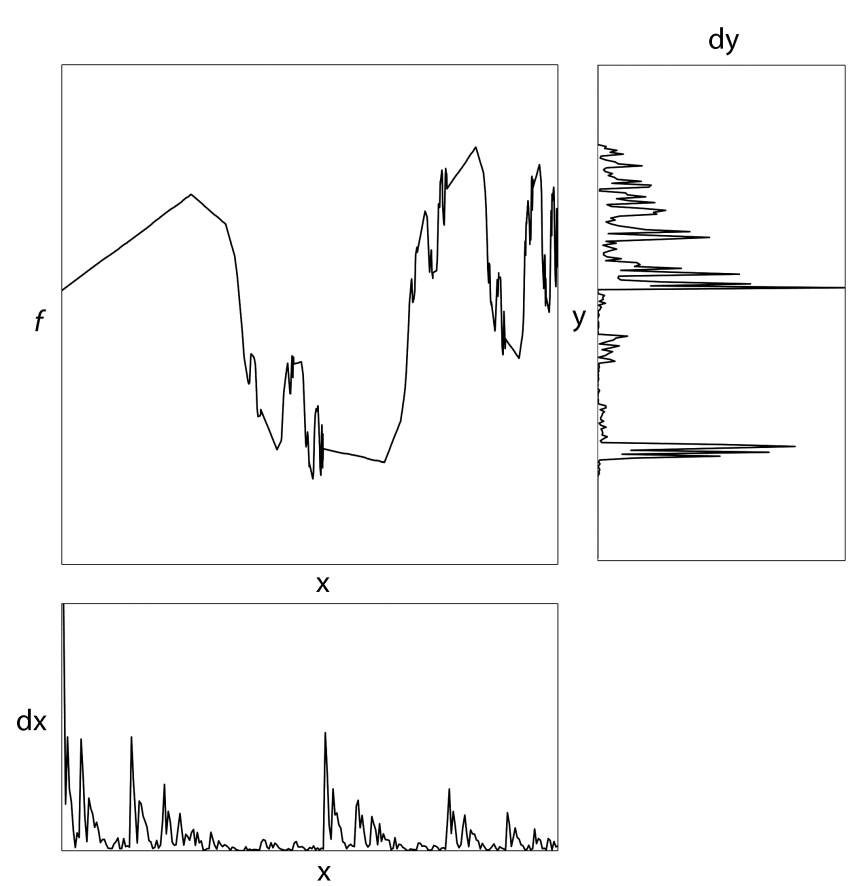

Fig. 1 The FM approach: from a multinomial multifractal measure, $d x$, to a complex derived measure, $d y$, via a fractal interpolating function, $f$, with equations given in the text. By construction, both $d x$ and $d y$ add up to one. Their scales are not shown due to aesthetic reasons. the graph $G$, the figure also displays the projections (histograms) $d x$ and $d y$, induced while carrying the previously mentioned chaos game according to a biased 51\%:8\%:41\% proportion on $w_{1}, w_{2}$, and $w_{3}$.

As the $x$-coordinate is independent of $y$, as implied by the zero value in Eq. (1), $d x$ turns out to be a recursively-generated deterministic (multinomial) multifractal, as given by a classical multiplicative cascade defined over $[0,1]$ and with length scales $(0.26,0.27$ and 0.47 , from the domain of the three mappings in $x$ ) and fixed multipliers (0.51, 0.08 and 0.41 , from the proportions used in the iterations), yielding a rather structured object containing an ordered sequence of spikes, in this case decaying in magnitude from left to right and with less mass in the middle subintervals, ad infinitum, due to the smaller multiplier. ${ }^{11}$

In turn, $d y$ is the derived distribution of $d x$ implied by $f$, and such a histogram is defined, for a given value of $y$, adding the corresponding $d x$ 's that satisfy $f(x)=y$. As can be seen in Fig. 1, the set $d y$, that is, the projection of the unique invariant measure generated by playing the chaos game over the $y$ axis, does not exhibit the same kind of repetition observed in $d x$. As may be inferred by the specific shape of the attracting wire $f, d y$ roughly follows the ups and downs of the wire-like function in terms of its $y$-crossings and the derived measure yields: (i) a sequence of rising events, from top to bottom, corresponding to oscillations on $f$ on the right-hand side of its domain and culminating with the highest peak that corresponds to the highest peak over $x$, (ii) a period of almost no activity over the middle and due to few crossings on such a region compounded with little mass over $x$, and (iii) an isolated "event" at the bottom whose mass arises from the juxtaposition of several oscillations on $f$ by the middle of its domain and hence the additions of several masses $d x$. It turns out, that the obtained $d y$ 's, for suitable combinations of the involved parameters, resemble complex natural time series such as rainfall patterns. ${ }^{7,12}$

As reflected by ubiquitous power-law scaling on their power spectra and the presence of a well-defined multifractal spectra, such deterministic sets typically do not exhibit obvious trends when the dimension of the fractal function $f$ does not approach its limiting value of two. Typically, they look like patterns of nature, and they appear random to the eye. ${ }^{5,14}$ To further illustrate such a fact, Fig. 2 shows an example of an attempt to fit a storm in Boston (shown on the far right) via the 


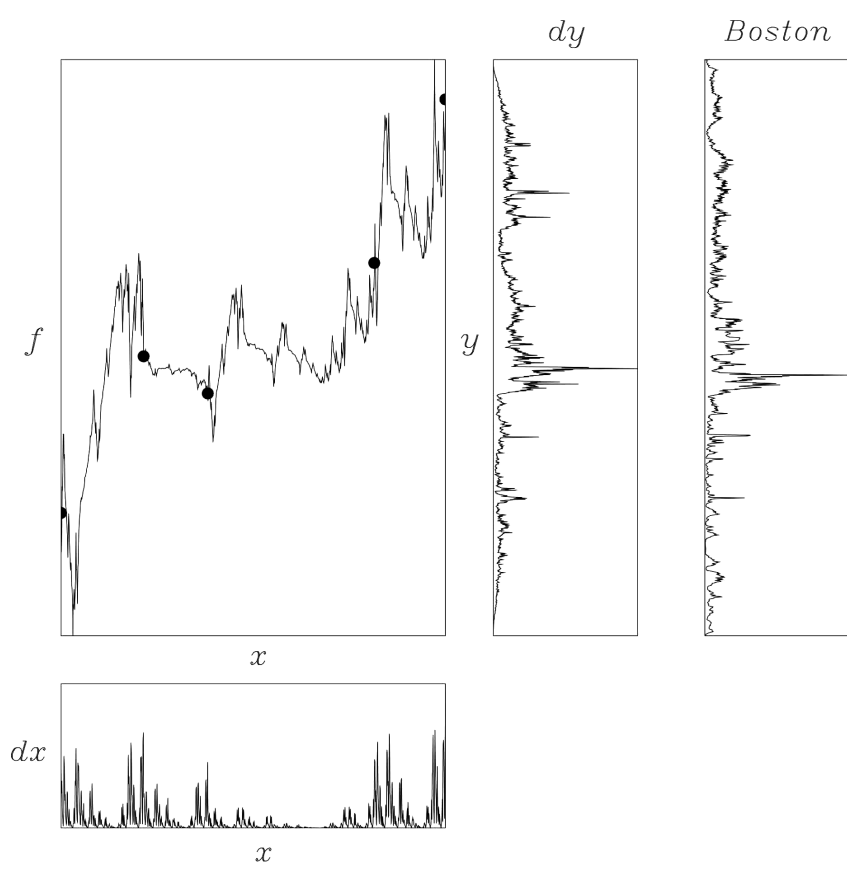

Fig. 2 An FM approximation of a Boston storm. The FM construction is based on five interpolating points and four affine maps. ${ }^{14}$ The Boston storm is shown normalized so that its values add up to one. Both the derived measure $d y$ and the rainfall intensities for the storm are defined over 1990 bins that correspond to measurements every 15 seconds.

fractal-multifractal approach using a fractal wire that passes by five points. As is seen, the projection $d y$, adjacent to the real set, looks similar in texture to the actual storm, which prompted even Benoit Mandelbrot, the late genius and father of fractals, to exclaim at a Gordon Conference on Fractals, "if the pattern on the right is rainfall, then the one on the left is also rainfall," to which the senior author of this article replied "thank you very much." In fact, the two sets shown are indistinguishable in regards to a host of statistical attributes, including a possible description of them based on notions of chaos theory. ${ }^{14}$

At the end, by varying the parameters defining the fractal function $f$ and the implied multinomial multifractal $d x$, one can obtain, via the chaos game, a plethora of interesting sets. ${ }^{5}$ Given the connection of a binomial multifractal with equal length scales and multipliers 0.7 and 0.3 with the observed energy dissipation in fully developed turbulence, ${ }^{15}$ in case $d x$ is such an input to a construction similar to the one given in Fig. 1 but based on two maps, the derived measure $d y$ may be given a "physical" interpretation as a transformation (via a fractal function $f$ ) of turbulence, or also, loosely speaking, such a $d y$ may be thought of as a "reflection" or a "projection" of turbulence. As illustrated in Fig. 1 and also Fig. 2 and as explained earlier, a measure $d y$ is built adding up values of $d x$ that satisfy $y=f(x)$. As a consequence, $d y$ is obtained performing a local "integration" over the fractal function, akin to a fractional integration, of the spiky multifractal $d x$ that comes from a multinomial multiplicative cascade.

\subsection{Extension via "Closing the Loop"}

As seen from Fig. 1, the structure of a fractal interpolating function is that of an open string that has a beginning and an end. In this manuscript, we explore the idea of closing a loop by having two fractal functions that meet at the ends. As an illustration, Fig. 3 shows a second wire $f_{2}$ of an obviously higher dimension, passing through the points $\{(0,0),(0.26,2.90),(0.53,-2.97),(1,1)\}$, which share the same start and end points as well as the $x$-values of the middle points of the wire in Fig. 1 named $f_{1}$. The three associated maps are

$$
\begin{aligned}
& \hat{w}_{1}\left(\begin{array}{l}
x \\
y
\end{array}\right)=\left(\begin{array}{ll}
0.26 & 0 \\
2.38 & 0.52
\end{array}\right)\left(\begin{array}{l}
x \\
y
\end{array}\right)+\left(\begin{array}{l}
0 \\
0
\end{array}\right), \\
& \hat{w}_{2}\left(\begin{array}{l}
x \\
y
\end{array}\right)=\left(\begin{array}{cc}
0.27 & 0 \\
-5.72 & -0.15
\end{array}\right)\left(\begin{array}{l}
x \\
y
\end{array}\right)+\left(\begin{array}{l}
0.26 \\
2.90
\end{array}\right),
\end{aligned}
$$

and

$$
\hat{w}_{3}\left(\begin{array}{l}
x \\
y
\end{array}\right)=\left(\begin{array}{cc}
0.47 & 0 \\
4.80 & -0.83
\end{array}\right)\left(\begin{array}{l}
x \\
y
\end{array}\right)+\left(\begin{array}{r}
0.53 \\
-2.97
\end{array}\right),
$$

corresponding to scalings $\hat{d}_{1}=0.52, \hat{d}_{2}=-0.15$, and $\hat{d}_{3}=-0.83$ and operate into the same intervals as before, i.e. $[0,0.26],[0.26,0.53],[0.53,1]$, according to the same weights, $51 \%: 8 \%: 41 \%$. This new $\hat{d y}$, named $d y 2$, is shown to the right of the previous $d y$ (as in Fig. 1), now called $d y 1$, and by combining the two, using $27 \%$ of the first and $73 \%$ of the second, we obtain the graph shown on the right, named dy_loop.

Of course, a curious reader would notice that the values employed in defining all the maps until now, i.e. Eqs. (4) to (9), are not entirely arbitrary, nor is the choice of the weights used to combine the projections from the two wires. In fact, the complex set shown in Fig. 3 will reappear later on in the paper when dealing with a specific storm gathered in Iowa City which resembles it. The interested reader may certainly change parameters via the online appendix located in http://puente. lawr.ucdavis.edu/omake/fractals2012.html in order 


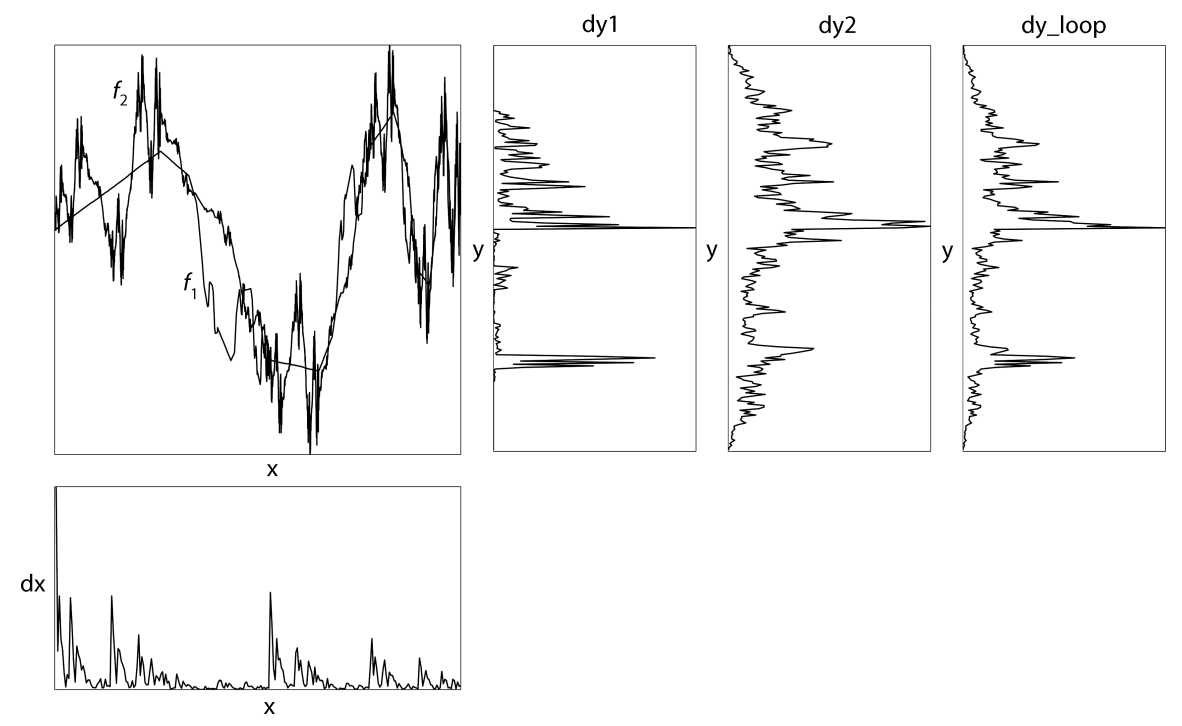

Fig. 3 Closing the loop via the FM approach: from a multinomial multifractal measure, $d x$, to two complex derived measures, $d y 1$ and $d y 2$, via fractal interpolating functions, $f_{1}$ and $f_{2}$, which weighed give a measure $d y \_l o o p$, with equations given in the text. By construction, all measures add up to one. Their scales are not shown due to aesthetic reasons.

to further appreciate the richness of the patterns that may be obtained by combining two wires.

As may be readily seen in Fig. 3 and also via the aforementioned online appendix, closing the loop provides interesting patterns that, although requiring more parameters, surely add to the repertoire that can be obtained via projections of fractal functions. After all, weighing two single wires using 0 's and 1's gives back the original fractalmultifractal approach and hence closing the loop results in a suitable generalization. In the remainder of the paper, we shall further illustrate these ideas to elucidate if with them we may closely encode four rainfall sets, as just accomplished via the original procedure. $^{12}$

\section{RESULTS AND DISCUSSION}

The data sets to be examined in this section are a subset (four) of seven storms in Iowa City gathered using an optical rain gauge. ${ }^{16}$ The four storms herein, labeled Iowa A through D per increasing number of major peaks, are selected for their distinct geometries. The storms took place on November 30th, October 3rd, November 1st, and May 3rd of 1990, corresponding to events that lasted 12.2, 9.8, 9.3, and 9.3 hours, respectively. The data were originally gathered every five seconds but are here slightly truncated to a multiple of 256 , downscaled to such a resolution, and finally normalized to unity. The new time intervals for the data points are, in order, every 2 minutes 40 seconds, 2 minutes 15 seconds, 2 minutes 10 seconds, and 2 minutes 10 seconds.

While other encodings of these sets for varying numbers of maps, based on the original FM approach and other extensions yielding attractors that are not functions, have been reported elsewhere, ${ }^{12}$ here we shall only concentrate on representations obtained with three maps (per wire), which, in accordance with Fig. 3, are representations utilizing 15 free parameters (two intermediate $x$ 's common to both wires, two intermediate $y$ 's per wire, three scalings $d_{n}$ per wire, two weights common to both wires, and a final weighing parameter used when combining the two derived histograms). In the interest of brevity, the parameters used in the upcoming figures are not included here, but in the online appendix at http://puente. lawr.ucdavis.edu/omake/fractals2012.html.

The results that follow are found by using a generalized particle swarm algorithm ${ }^{17}$ to minimize the $l^{2}$-norm of averaged cumulative distribution deviations between the loopy fit and the data,

$$
\text { err }=\sqrt{\frac{1}{N_{0}}} \sum_{n=1}^{N_{0}}\left(c_{n}-\hat{c}_{n}\right)^{2},
$$

where $N_{0}$ is the number of data points (256 in this case), and $c_{n}$ and $\hat{c}_{n}$ denote the $n$th value of the cumulative distribution of the original record and the generated fit, respectively. To ensure geometric similarity between plausible solutions and the original data set, we impose a penalty on the objective 

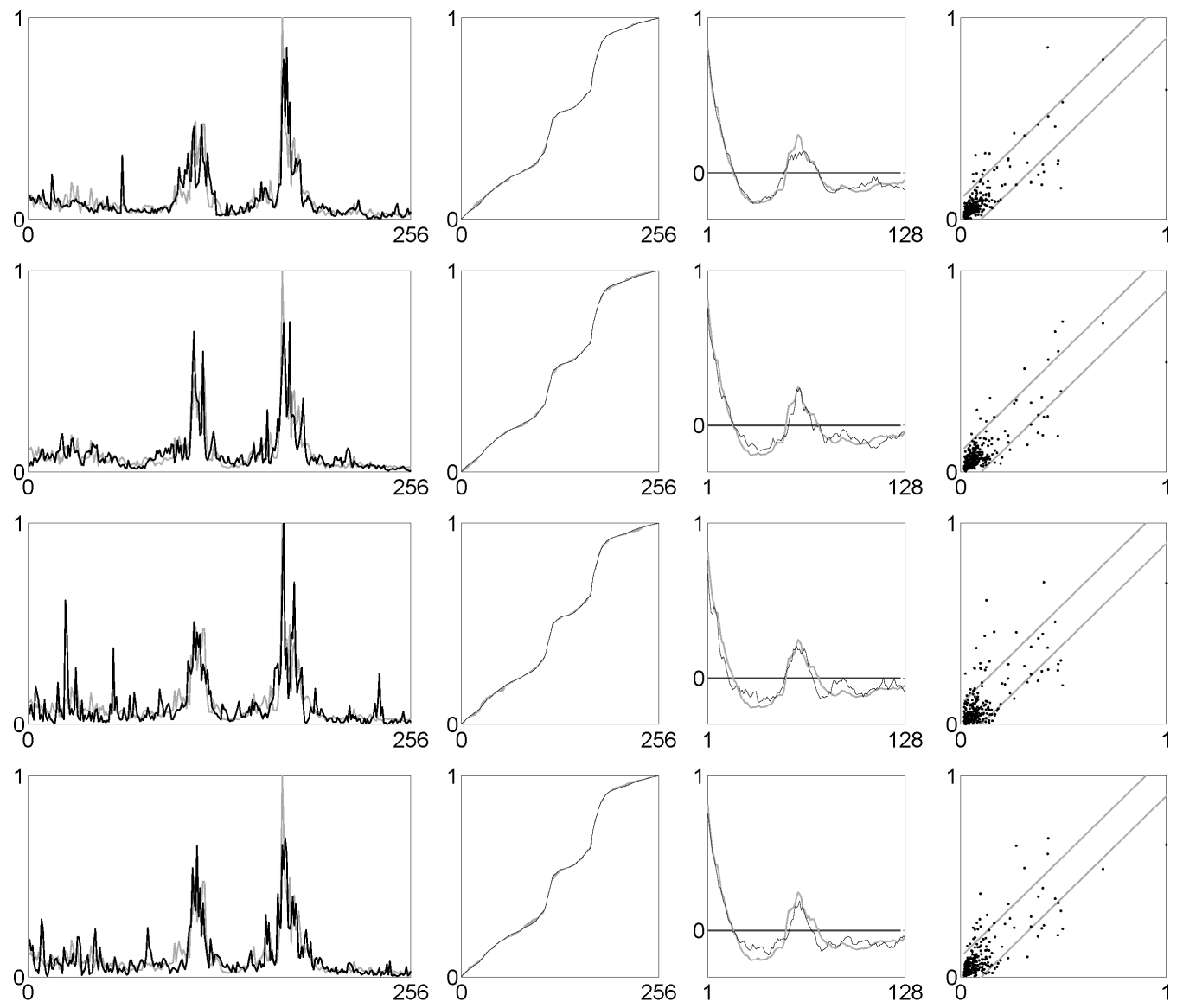

Fig. 4 Iowa A renderings. Left column: the fit, in black, imposed on the original data, in gray. First middle column: the cumulative distributions of the data and the fit, with errors colored as area between the curves. Second middle column: the autocorrelation function of the data and the fit. Right column: scatterplot of the data (horizontal) vs. the fit (vertical). Diagonal lines indicate $\pm 10 \%$ lines. The representations correspond to ErrCumul in Table 1, from top to bottom. Readers interested in reconstructing the plots may navigate to the online Appendix, where all parameters may be found: http://puente.lawr.ucdavis.edu/omake/fractals2012.html.

Table 1 Statistics for the Iowa A Storm Representations: $l^{2}$-Norm of Averaged Error on Data (ErrData), $l^{2}$-Norm of Averaged Cumulative Distribution Deviations (ErrCuml), Maximum Deviation between Cumulative Distributions (MaxDev), Nash-Sutcliffe Model Efficiency Coefficient for the Data (NS-Data), Nash-Sutcliffe Coefficient for the Autocorrelation (NS-Auto), Fractal Dimension (FractalD), Entropy Dimension (EntropyD), Autocorrelation Lag at 1/e (Lag 1/e), and Autocorrelation at Lag 0 (Lag 0$)$. The Representations are All Achieved Using 15 Parameters.

\begin{tabular}{cccccccccc}
\hline$\#$ & ErrData & ErrCuml & MaxDev & NS-Data & NS-Auto & FractalD & EntropyD & Lag 1/e & Lag 0 \\
\hline (Data) & $\mathrm{n} / \mathrm{a}$ & $\mathrm{n} / \mathrm{a}$ & $\mathrm{n} / \mathrm{a}$ & $\mathrm{n} / \mathrm{a}$ & $\mathrm{n} / \mathrm{a}$ & 1.33 & 0.91 & 9 & 18 \\
1 & $0.28 \%$ & $0.72 \%$ & $2.27 \%$ & 0.64 & 0.96 & 1.33 & 0.92 & 7 & 18 \\
2 & $0.28 \%$ & $0.76 \%$ & $1.84 \%$ & 0.64 & 0.93 & 1.37 & 0.91 & 7 & 18 \\
3 & $0.36 \%$ & $0.77 \%$ & $2.10 \%$ & 0.43 & 0.83 & 1.38 & 0.90 & 8 & 15 \\
4 & $0.32 \%$ & $0.77 \%$ & $1.96 \%$ & 0.56 & 0.90 & 1.43 & 0.91 & 7 & 17 \\
\hline
\end{tabular}

function if the maximum deviations in cumulative distributions exceed $10 \%$ anywhere.

In Figs. 4-7, we see four local minima results per each of the storm sets corresponding to the column ErrCuml (i.e. Eq. (10)) in Tables 1-4, listed in increasing order. In each figure, the plots on the left are the best fits for the set (in dark) imposed on top of the set itself (in gray), which are rescaled 

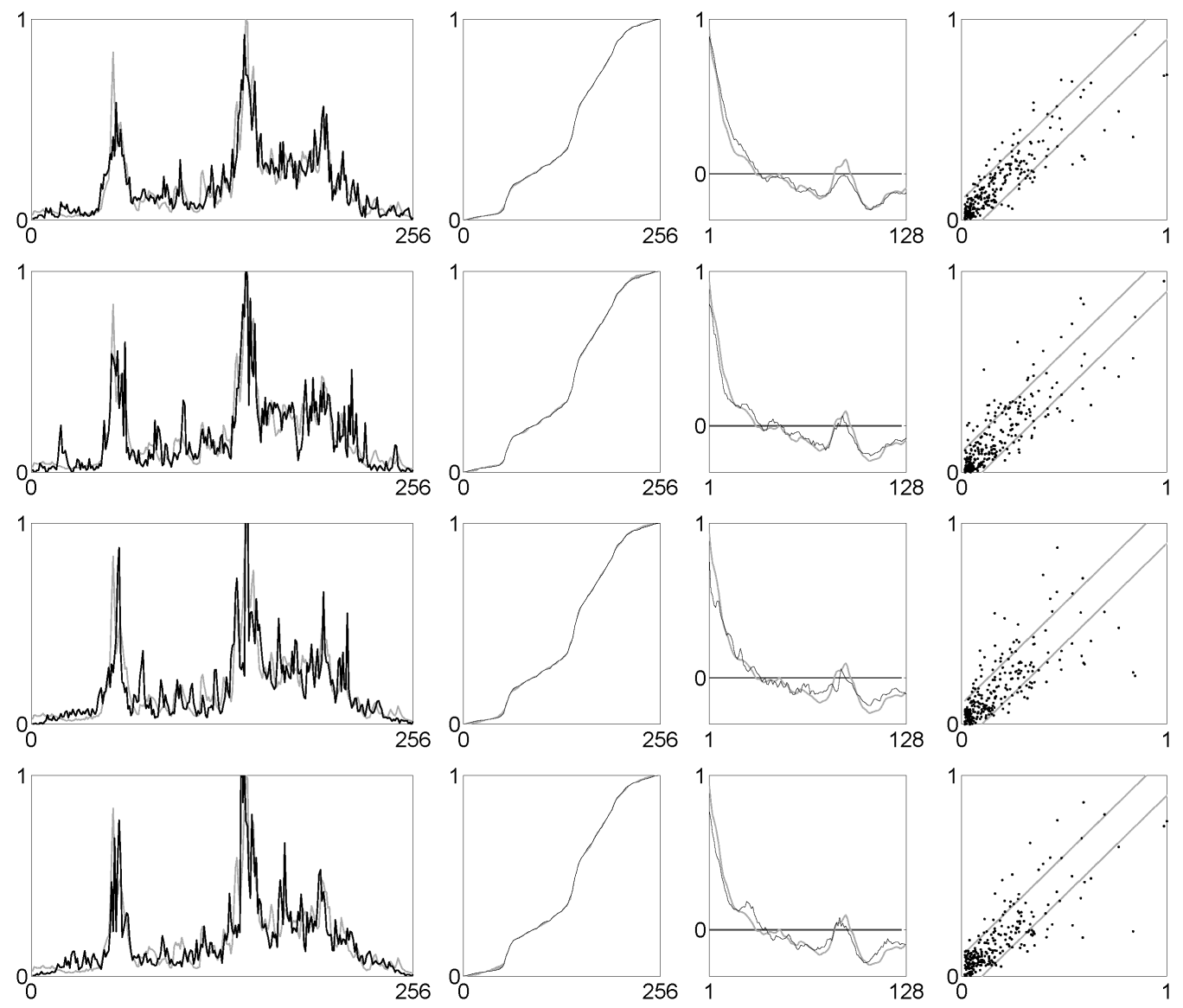

Fig. 5 Iowa $B$ renderings. The row and column setup is as in Fig. 4. From top to bottom, the representations correspond to ErrCumul in Table 2.

Table 2 Statistics for the Iowa B Storm Representations. Column Descriptions are as in Table 1. The Representations are All Achieved Using 15 Parameters.

\begin{tabular}{cccccccccc}
\hline$\#$ & ErrData & ErrCuml & MaxDev & NS-Data & NS-Auto & FractalD & EntropyD & Lag 1/e & Lag 0 \\
\hline (Data) & $\mathrm{n} / \mathrm{a}$ & $\mathrm{n} / \mathrm{a}$ & $\mathrm{n} / \mathrm{a}$ & $\mathrm{n} / \mathrm{a}$ & $\mathrm{n} / \mathrm{a}$ & 1.36 & 0.94 & 10 & 31 \\
1 & $0.19 \%$ & $0.48 \%$ & $1.42 \%$ & 0.77 & 0.97 & 1.42 & 0.95 & 12 & 33 \\
2 & $0.21 \%$ & $0.61 \%$ & $1.54 \%$ & 0.71 & 0.94 & 1.43 & 0.94 & 8 & 35 \\
3 & $0.26 \%$ & $0.65 \%$ & $1.66 \%$ & 0.56 & 0.87 & 1.37 & 0.94 & 10 & 35 \\
4 & $0.25 \%$ & $0.67 \%$ & $1.81 \%$ & 0.57 & 0.92 & 1.36 & 0.94 & 9 & 36 \\
\hline
\end{tabular}

for presentational purposes such that the maximum value of the original data is 1 . To their right are plots of the cumulative distributions, with that of the data set shown as a black line and deviations shown as gray areas. Next are the autocorrelation functions of the fit (dark) on top of the data (gray). The right-most plot illustrates the scatter of the data ( $x$-axis) versus the fits ( $y$-axis); the two gray bands denote $\pm 10 \%$ deviation from the one-to-one line. As a side note, the bottom result in Fig. 5 is the same representation used to illustrate the loopy idea in Figs. 1 and 3.
Evidently, these renderings are terrific fits for the data sets, not only in their distributions (intentionally optimized) but also in their autocorrelation functions. Notice that the placement of the major peaks are well preserved, with the majority of points falling within the $10 \%$ bands, and for the first three storms, any outliers in the scatterplots do not stray particularly far from the center.

The goodness of the results can also be verified by considering the information in Tables 1-4. First of all, notice that the objective function values (ErrCuml) are all less than $0.80 \%$ (and does 

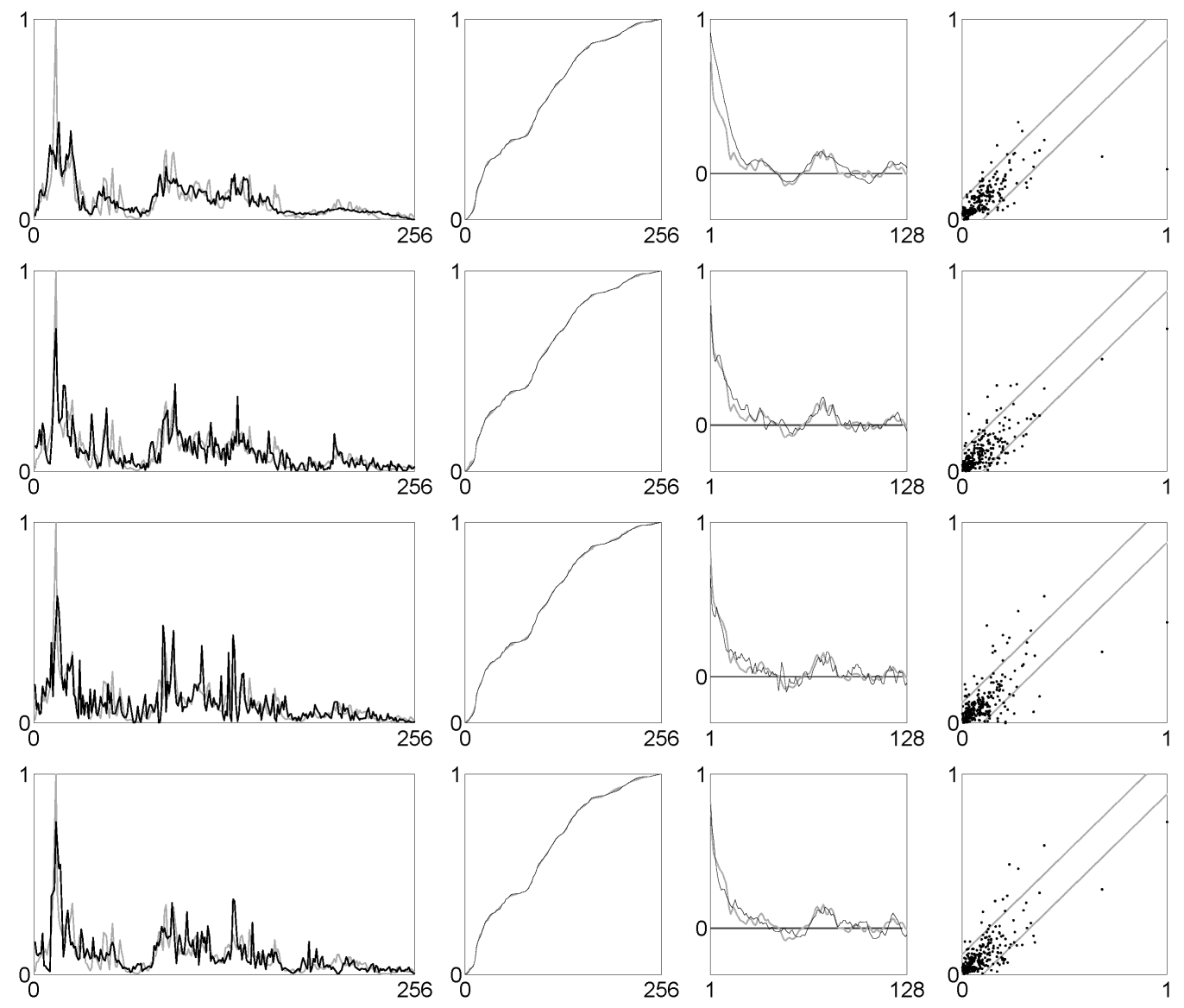

Fig. 6 Iowa $C$ renderings. The row and column setup is as in Fig. 4. From top to bottom, the representations correspond to ErrCumul in Table 3.

Table 3 Statistics for the Iowa C Storm Representations. Column Descriptions are as in Table 1. The Representations are all Achieved Using 15 Parameters.

\begin{tabular}{cccccccccc}
\hline$\#$ & ErrData & ErrCuml & MaxDev & NS-Data & NS-Auto & FractalD & EntropyD & Lag 1/e & Lag 0 \\
\hline (Data) & $\mathrm{n} / \mathrm{a}$ & $\mathrm{n} / \mathrm{a}$ & $\mathrm{n} / \mathrm{a}$ & $\mathrm{n} / \mathrm{a}$ & $\mathrm{n} / \mathrm{a}$ & 1.37 & 0.93 & 8 & 46 \\
1 & $0.29 \%$ & $0.62 \%$ & $2.38 \%$ & 0.51 & 0.83 & 1.39 & 0.94 & 13 & 44 \\
2 & $0.27 \%$ & $0.64 \%$ & $2.25 \%$ & 0.59 & 0.91 & 1.42 & 0.94 & 9 & 36 \\
3 & $0.34 \%$ & $0.64 \%$ & $2.18 \%$ & 0.34 & 0.82 & 1.46 & 0.93 & 7 & 45 \\
4 & $0.28 \%$ & $0.66 \%$ & $2.07 \%$ & 0.55 & 0.88 & 1.42 & 0.93 & 6 & 39 \\
\hline
\end{tabular}

not exceed $2.40 \%$ anywhere, i.e. MaxDev) and the $l^{2}$-norm of averaged errors on the data set itself (ErrData; not optimized) are less than $0.40 \%$. This is confirmed by the large Nash-Sutcliffe model efficiency coefficients (NS-Data and NS-Auto; calculated as 1 minus the ratio of the sum of squared deviations between data and model to the sum of squared deviations of the data relative to its mean) between the individual data points and also the autocorrelation functions, and moreover, by the close fittings of the fractal and entropy dimensions and lags of the autocorrelation functions at 1 /e and 0 in the last four columns.
As verifiable from the parameters given in the online Appendix, these imperfect but nonetheless remarkable results come from local minima in distinct regions of parameter space as identified by the particle swarm procedure, which implies that a unique global solution, if one exists at all, may be difficult to find. This also happens to be the case for similar results computed based on four maps (also found in the Appendix), which yield results similar to the ones presented here.

As may be appreciated, the first three storms were better fitted than the last, whose additional scatter is reflected by the lower Nash-Sutcliffe 

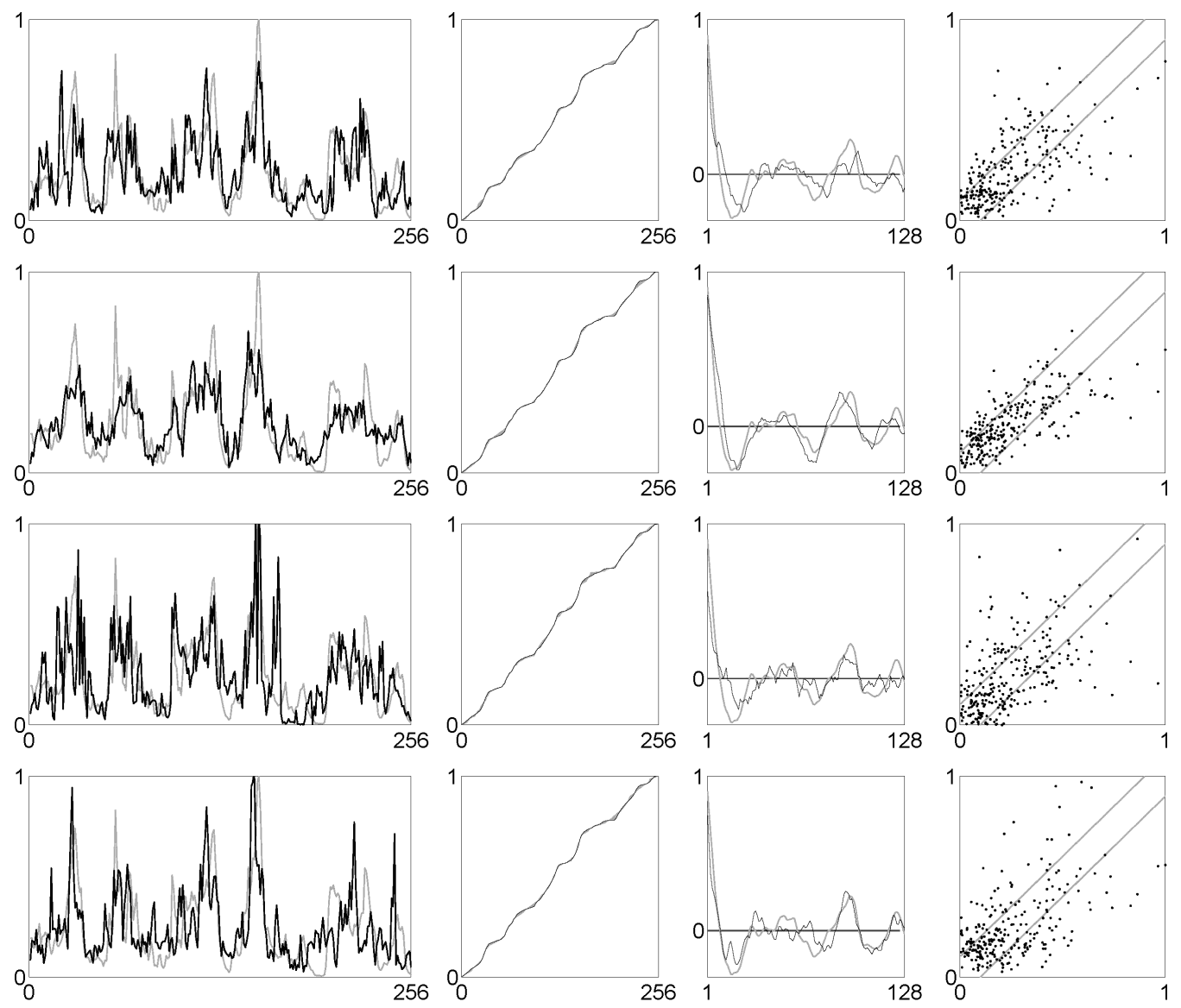

Fig. 7 Iowa $D$ renderings. The row and column setup is as in Fig. 4. From top to bottom, the representations correspond to ErrCumul in Table 4.

Table 4 Statistics for the Iowa D Storm Representations. Column Descriptions are as in Table 1. The Representations are All Achieved Using 15 Parameters.

\begin{tabular}{cccccccccc}
\hline$\#$ & ErrData & ErrCuml & MaxDev & NS-Data & NS-Auto & FractalD & EntropyD & Lag 1/e & Lag 0 \\
\hline (Data) & n/a & n/a & n/a & n/a & n/a & 1.48 & 0.96 & 5 & 9 \\
1 & $0.24 \%$ & $0.73 \%$ & $2.33 \%$ & 0.36 & 0.42 & 1.56 & 0.97 & 4 & 13 \\
2 & $0.21 \%$ & $0.73 \%$ & $1.95 \%$ & 0.51 & 0.81 & 1.54 & 0.98 & 7 & 12 \\
3 & $0.27 \%$ & $0.74 \%$ & $1.85 \%$ & 0.15 & 0.08 & 1.51 & 0.96 & 4 & 11 \\
4 & $0.27 \%$ & $0.77 \%$ & $2.26 \%$ & 0.18 & 0.65 & 1.49 & 0.96 & 3 & 10 \\
\hline
\end{tabular}

coefficients of data and autocorrelation (relative to the other fits) despite the excellent preservation of the fractal and autocorrelation qualifiers (see Table 4). Notice, however, that as actual data sets are not free of measurement errors, ${ }^{18}$ these fits can be considered sufficiently reasonable encodings, or at the very least, very suitable simulations for what nature produces. For after all, without knowing what the real data set is, no one can distinguish between the real data set and the FM projections, and the quality of the fits remains when the data is scaled back to the original resolution.
For the sake of comparison, it must be emphasized that the excellent results herein are as good, but not better, as those that may be obtained from a single wire and other extensions of the fractalmultifractal approach that do not close the loop and that resulted in other fits requiring from nine to 19 parameters. ${ }^{12}$ As closing the loop requires 15 parameters, the representations herein are indeed suitable deterministic alternatives for the encoding of the rainfall sets.

As may be verified on the online Appendix, having additional parameters in the search leads to 
combinations of wires and not to the usage of single ones and such implies that there are multiple approximations for a given pattern within the framework of projections and that the involved fitness surface is difficult to navigate towards a global optima. Overall, the results herein support the notion that simplicity may be at the root of complexity, in this case via projections and without the need of invoking random mechanisms. ${ }^{19}$

\section{CONCLUSIONS}

We have demonstrated how combining two projections of fractal interpolating functions sharing a common multinomial multifractal input reasonably generalizes the FM method, which results in suitable encodings of natural patterns. Although the extension requires more parameters than the original approach, we obtain in return additional flexibility in the projections; that is, the second projection can now account for textures that the first projection may have missed, which then leads to a better fit. All of this is accomplished while maintaining parsimony in the parameter count, as evidenced by the compression ratios of $17: 1$ for 256 -value sets and in excess of 440:1 for the original sets.

\section{REFERENCES}

1. I. Rodríguez-Iturbe, Scale of fluctuation of rainfall models, Water Resour. Res. 22(9) (1986) 15S-37S.

2. S. Lovejoy and D. Schertzer, Multifractals, universality classes and satellite and radar measurements of cloud and rain fields, J. Geophys. Res. 95(D3) (1990) 2021-2034.

3. C. E. Puente, A new approach to hydrologic modeling: Derived distributions revisited, J. Hydrol. 18 (1996) 65-80.

4. M. F. Barnsley, Fractals Everywhere (Academic Press, San Diego, 1988).

5. C. E. Puente, A universe of projections: May Plato be right? Chaos Solitons Fractals 19(2) (2004) 241253.

6. N. Obregón, C. E. Puente and B. Sivakumar, Modeling high resolution rain rates via a deterministic fractal-multifractal approach, Fractals 10(3) (2002) 387-394.
7. N. Obregón, B. Sivakumar and C. E. Puente, A deterministic geometric representation of temporal rainfall. Sensitivity analysis for a storm in Boston, J. Hydrol. 269(3-4) (2002) 224-235.

8. C. E. Puente and B. Sivakumar, A deterministic width function model, Nonlin Process. Geophys. 10(6) (2003) 525-529.

9. A. Cortis, C. E. Puente and B. Sivakumar, Nonlinear extensions of a fractal-multifractal approach for environmental modeling, Stoch. Environ. Res. Risk Assess. 23(7) (2009) 897-906.

10. A. Cortis, C. E. Puente and B. Sivakumar, Encoding hydrologic information via a fractal geometric approach and its extensions, Stoch. Environ. Res. Risk Assess. 24(5) (2010) 625-632.

11. B. B. Mandelbrot, Multifractal measures especially for the geophysicist. In Fractals in Geophysics, eds. C. H. Scholz and B. B. Mandelbrot (BirkhauserVerlag, Basel, 1989), pp. 1-42.

12. H. Huang, A. Cortis and C. E. Puente, Geometric harnessing of precipitation records: Reexamining four storms from Iowa City, Stoch. Environ. Res. Risk Assess. (2012), DOI: 10.1007/s00477-0120617-6.

13. C. E. Puente and N. Obregón, A geometric Platonic approach to multifractality and turbulence, Fractals 7(4) (1999) 403-420.

14. C. E. Puente and N. Obregón, A deterministic geometric representation of temporal rainfall. Results for a storm in Boston, Water Resour. Res. 32(9) (1996) 2825-2839.

15. C. Meneveau and K. R. Sreenivasan, Simple multifractal cascade model for fully developed turbulence, Phys. Rev. Lett. 59 (1987) 1424-1427.

16. K. P. Georgakakos, A. A. Carsteanu, P. L. Sturdevant and J. A. Cramer, Observation and analysis of Midwestern rain rates, J. Appl. Meteor. 33(12) (1994) 1433-1444.

17. J. L. Fernández-Martínez, E. García Gonzalo, J. P. Fernández-Álvarez, H. A. Kuzma and C. O. Menéndez Pérez, PSO: A powerful algorithm to solve geophysical inverse problems. Application to a 1D-DC resistivity case, J. Appl. Geophys. 71(1) (2010) 13-25.

18. L. G. Lanza, E. Vuerich and I. Gnecco, Analysis of highly accurate rain intensity measurements from a field test site, Adv. Geosci. 25 (2010) 37-44.

19. C. E. Puente and B. Sivakumar, Modeling hydrologic complexity: A case for geometric determinism, Hydrol. Earth Syst. Sci. 11 (2007) 721-724. 